\title{
BROWDER'S TYPE STRONG CONVERGENCE THEOREMS FOR INFINITE FAMILIES OF NONEXPANSIVE MAPPINGS IN BANACH SPACES
}

\author{
TOMONARI SUZUKI
}

Received 19 August 2005; Revised 24 February 2006; Accepted 26 February 2006

We prove Browder's type strong convergence theorems for infinite families of nonexpansive mappings. One of our main results is the following: let $C$ be a bounded closed convex subset of a uniformly smooth Banach space $E$. Let $\left\{T_{n}: n \in \mathbb{N}\right\}$ be an infinite family of commuting nonexpansive mappings on $C$. Let $\left\{\alpha_{n}\right\}$ and $\left\{t_{n}\right\}$ be sequences in $(0,1 / 2)$ satisfying $\lim _{n} t_{n}=\lim _{n} \alpha_{n} / t_{n}^{\ell}=0$ for $\ell \in \mathbb{N}$. Fix $u \in C$ and define a sequence $\left\{u_{n}\right\}$ in $C$ by $u_{n}=\left(1-\alpha_{n}\right)\left(\left(1-\sum_{k=1}^{n} t_{n}^{k}\right) T_{1} u_{n}+\sum_{k=1}^{n} t_{n}^{k} T_{k+1} u_{n}\right)+\alpha_{n} u$ for $n \in \mathbb{N}$. Then $\left\{u_{n}\right\}$ converges strongly to $P u$, where $P$ is the unique sunny nonexpansive retraction from $C$ onto $\bigcap_{n=1}^{\infty} F\left(T_{n}\right)$.

Copyright (c) 2006 Tomonari Suzuki. This is an open access article distributed under the Creative Commons Attribution License, which permits unrestricted use, distribution, and reproduction in any medium, provided the original work is properly cited.

\section{Introduction}

Let $C$ be a closed convex subset of a Banach space $E$. A mapping $T$ on $C$ is called a nonexpansive mapping if $\|T x-T y\| \leq\|x-y\|$ for all $x, y \in C$. We denote by $F(T)$ the set of fixed points of $T$. We know that $F(T)$ is nonempty in the case that $E$ is uniformly smooth and $C$ is bounded; see Baillon [1]. When $E$ has the Opial property and $C$ is weakly compact, $F(T)$ is also nonempty; see $[11,13]$. See also $[4,5,10]$ and others. Fix $u \in C$. Then for each $\alpha \in(0,1)$, there exists a unique point $x_{\alpha}$ in $C$ satisfying $x_{\alpha}=(1-\alpha) T x_{\alpha}+\alpha u$ because the mapping $x \mapsto(1-\alpha) T x+\alpha u$ is contractive; see [2]. In 1967 Browder [6] proved the following strong convergence theorem.

Theorem 1.1 (Browder [6]). Let $C$ be a bounded closed convex subset of a Hilbert space $E$ and let $T$ be a nonexpansive mapping on $C$. Let $\left\{\alpha_{n}\right\}$ be a sequence in $(0,1)$ converging to 0 . Fix $u \in C$ and define a sequence $\left\{u_{n}\right\}$ in $C$ by

$$
u_{n}=\left(1-\alpha_{n}\right) T u_{n}+\alpha_{n} u
$$

for $n \in \mathbb{N}$. Then $\left\{u_{n}\right\}$ converges strongly to the element of $F(T)$ nearest to $u$. 
Reich extended this theorem to uniformly smooth Banach spaces in [17]. Using the notion of Bochner integral and (invariant) mean, Shioji and Takahashi in [18] proved Browder's type strong convergence theorems for families of nonexpansive mappings

Very recently, the author proved the following Browder's type strong convergence theorem for one-parameter nonexpansive semigroups. This is a generalization of the results in $[19,25]$. We remark that we do not use the notion of Bochner integral.

Theorem 1.2 [24]. Let $C$ be a weakly compact convex subset of a Banach space E. Assume that either of the following holds:

(i) E is uniformly convex with uniformly Gâteaux differentiable norm;

(ii) $E$ is uniformly smooth; or

(iii) $E$ is a smooth Banach space with the Opial property and the duality mapping $J$ of $E$ is weakly sequentially continuous at zero.

Let $\{T(t): t \geq 0\}$ be a one-parameter nonexpansive semigroup on $C$. Let $\tau$ be a nonnegative real number. Let $\left\{\alpha_{n}\right\}$ and $\left\{t_{n}\right\}$ be sequences of real numbers satisfying $0<\alpha_{n}<1,0<\tau+t_{n}$ and $t_{n} \neq 0$ for $n \in \mathbb{N}$, and $\lim _{n} t_{n}=\lim _{n} \alpha_{n} / t_{n}=0$. Fix $u \in C$ and define a sequence $\left\{u_{n}\right\}$ in C by

$$
u_{n}=\left(1-\alpha_{n}\right) T\left(\tau+t_{n}\right) u_{n}+\alpha_{n} u
$$

for $n \in \mathbb{N}$. Then $\left\{u_{n}\right\}$ converges strongly to $P u$, where $P$ is the unique sunny nonexpansive retraction from $C$ onto $\bigcap_{t \geq 0} F(T(t))$.

Also, very recently, the author proved Krasnoselskii and Mann's type convergence theorems for infinite families of nonexpansive mappings in [21]. See also [20]. In this paper, using the idea in [21], we prove Browder's type strong convergence theorems for infinite families of nonexpansive mappings without assuming the strict convexity of the Banach space. We remark that if we assume the strict convexity, its proof is very easy because the set of common fixed points of countable families of nonexpansive mappings is the set of fixed points of some single nonexpansive mapping; see Bruck [8]. We also remark that we do not use the notion of (invariant) mean.

\section{Preliminaries}

Throughout this paper, we denote by $\mathbb{N}, \mathbb{Z}, \mathbb{Q}$, and $\mathbb{R}$ the set of all positive integers, all integers, all rational numbers, and all real numbers, respectively.

Let $\left\{x_{n}\right\}$ be a sequence in a topological space $X$. By the axiom of choice, there exist a directed set $(D, \leq)$ and a universal subnet $\left\{x_{f(v)}: \nu \in D\right\}$ of $\left\{x_{n}\right\}$, that is,

(i) $f$ is a mapping from $D$ into $\mathbb{N}$ such that for each $n \in \mathbb{N}$ there exists $\nu_{0} \in D$ such that $\nu \geq \nu_{0}$ implies $f(\nu) \geq n$;

(ii) for each subset $A$ of $X$, there exists $\nu_{0} \in D$ such that either $\left\{x_{f(\nu)}: \nu \geq \nu_{0}\right\} \subset A$ or $\left\{x_{f(v)}: v \geq v_{0}\right\} \subset X \backslash A$ holds.

In this paper, we often use $\left\{x_{v}: \nu \in D\right\}$ instead of $\left\{x_{f(v)}: v \in D\right\}$, for short. We know that if a net $\left\{x_{\nu}\right\}$ is universal and $g$ is a mapping from $X$ into an arbitrary set $Y$, then $\left\{g\left(x_{\nu}\right)\right\}$ is also universal. We also know that if $X$ is compact, then a universal net $\left\{x_{\nu}\right\}$ always converges. See [12] for details. 
Let $E$ be a real Banach space. We denote by $E^{*}$ the dual of $E$. $E$ is called uniformly convex if for each $\varepsilon>0$, there exists $\delta>0$ such that $\|x+y\| / 2<1-\delta$ for all $x, y \in E$ with $\|x\|=\|y\|=1$ and $\|x-y\| \geq \varepsilon$. $E$ is said to be smooth or said to have a Gâteaux differentiable norm if the limit

$$
\lim _{t \rightarrow 0} \frac{\|x+t y\|-\|x\|}{t}
$$

exists for each $x, y \in E$ with $\|x\|=\|y\|=1$. $E$ is said to have a uniformly Gâteaux differentiable norm if for each $y \in E$ with $\|y\|=1$, the limit is attained uniformly in $x \in E$ with $\|x\|=1$. E is said to be uniformly smooth or said to have a uniformly Fréchet differentiable norm if the limit is attained uniformly in $x, y \in E$ with $\|x\|=\|y\|=1$. E is said to have the Opial property [14] if for each weakly convergent sequence $\left\{x_{n}\right\}$ in $E$ with weak limit $x_{0}$,

$$
\liminf _{n \rightarrow \infty}\left\|x_{n}-x_{0}\right\|<\liminf _{n \rightarrow \infty}\left\|x_{n}-x\right\|
$$

holds for all $x \in E$ with $x \neq x_{0}$. We remark that we may replace "liminf" by "limsup." That is, $E$ has the Opial property if and only if for each weakly convergent sequence $\left\{x_{n}\right\}$ in $E$ with weak limit $x_{0}$,

$$
\limsup _{n \rightarrow \infty}\left\|x_{n}-x_{0}\right\|<\limsup _{n \rightarrow \infty}\left\|x_{n}-x\right\|
$$

holds for all $x \in E$ with $x \neq x_{0}$.

Let $E$ be a smooth Banach space. The duality mapping $J$ from $E$ into $E^{*}$ is defined by

$$
\langle x, J(x)\rangle=\|x\|^{2}=\|J(x)\|^{2}
$$

for all $x \in E$. $J$ is said to be weakly sequentially continuous at zero if for every sequence $\left\{x_{n}\right\}$ in $E$ which converges weakly to $0 \in E,\left\{J\left(x_{n}\right)\right\}$ converges weakly* to $0 \in E^{*}$.

A convex subset $C$ of a Banach space $E$ is said to have normal structure [3] if for every bounded convex subset $K$ of $C$ which contains more than one point, there exists $z \in K$ such that

$$
\sup _{x \in K}\|x-z\|<\sup _{x, y \in K}\|x-y\| \text {. }
$$

We know that compact convex subsets of any Banach spaces and closed convex subsets of uniformly convex Banach spaces have normal structure. Turett [27] proved that uniformly smooth Banach spaces have normal structure. Also, Gossez and Lami Dozo [11] proved that every weakly compact convex subset of a Banach space with the Opial property has normal structure. We recall that a closed convex subset $C$ of a Banach space $E$ is said to have the fixed point property for nonexpansive mappings (FPP, for short) if for every bounded closed convex subset $K$ of $C$, every nonexpansive mapping on $K$ has a fixed point. So, by Kirk's fixed point theorem [13], every weakly compact convex subset with normal structure has FPP. 
Let $C$ and $K$ be subsets of a Banach space $E$. A mapping $P$ from $C$ into $K$ is called sunny [7] if

$$
P(P x+t(x-P x))=P x
$$

for $x \in C$ with $P x+t(x-P x) \in C$ and $t \geq 0$. The following is proved in [15].

Lemma 2.1 (Reich [15]). Let $E$ be a smooth Banach space and let $C$ be a convex subset of $E$. Let $K$ be a subset of $C$ and let $P$ be a retraction from $C$ onto $K$. Then the following are equivalent:

(i) $\langle x-P x, J(P x-y)\rangle \geq 0$ for all $x \in C$ and $y \in K$;

(ii) $P$ is both sunny and nonexpansive.

Hence, there is at most one sunny nonexpansive retraction from $C$ onto $K$.

The following lemma is proved in [24]. However, it is essentially proved in [16]. See also [26].

Lemma 2.2 (Reich [16]). Let $C$ be a nonempty closed convex subset of a Banach space $E$ with a uniformly Gâteaux differentiable norm. Let $\left\{x_{\alpha}: \alpha \in D\right\}$ be a net in $E$ and let $z \in C$. Suppose that the limits of $\left\{\left\|x_{\alpha}-y\right\|\right\}$ exist for all $y \in C$. Then the following are equivalent:

(i) $\lim _{\alpha \in D}\left\|x_{\alpha}-z\right\|=\min _{y \in C} \lim _{\alpha \in D}\left\|x_{\alpha}-y\right\|$;

(ii) $\lim \sup _{\alpha \in D}\left\langle y-z, J\left(x_{\alpha}-z\right)\right\rangle \leq 0$ for all $y \in C$;

(iii) $\liminf _{\alpha \in D}\left\langle y-z, J\left(x_{\alpha}-z\right)\right\rangle \leq 0$ for all $y \in C$.

The following lemma is well known.

Lemma 2.3. Let $\left\{u_{n}\right\}$ be a sequence in a Banach space $E$ and let $z$ belong to E. Assume that every subsequence $\left\{u_{n_{i}}\right\}$ of $\left\{u_{n}\right\}$ has a subsequence converging to $z$. Then $\left\{u_{n}\right\}$ itself converges to $z$.

From Lemma 2.3, we obtain the following.

Lemma 2.4. Let $\left\{u_{n}\right\}$ be a sequence in a Banach space E. Assume that $\left\{u_{n}\right\}$ has at most one cluster point, and every subsequence of $\left\{u_{n}\right\}$ has a cluster point. Then $\left\{u_{n}\right\}$ converges.

Proof. Since $\left\{u_{n}\right\}$ is a subsequence of $\left\{u_{n}\right\},\left\{u_{n}\right\}$ has a cluster point $z \in E$. Let $\left\{u_{n_{i}}\right\}$ be an arbitrary subsequence of $\left\{u_{n}\right\}$. Then by assumption $\left\{u_{n_{i}}\right\}$ has a cluster point $w \in E$. Since $w$ is also a cluster point of $\left\{u_{n}\right\}$, we have $w=z$. Hence, $\left\{u_{n_{i}}\right\}$ has a cluster point $z \in E$. That is, there exists a subsequence of $\left\{u_{n_{i}}\right\}$ converging to $z$. So, by Lemma 2.3, $\left\{u_{n}\right\}$ converges to $z$. This completes the proof.

\section{Fixed point theorem}

The following theorem is one of the most famous fixed point theorems for families of nonexpansive mappings.

Theorem 3.1 (Bruck [9]). Suppose a closed convex subset $C$ of a Banach space E has the fixed point property for nonexpansive mappings, and $C$ is either weakly compact, or bounded and separable. Then for any commuting family $S$ of nonexpansive mappings on $C$, the set of common fixed points of $S$ is a nonempty nonexpansive retract of $C$. 
Using Theorem 3.1, we prove the following fixed point theorem.

Theorem 3.2. Let $C$ be a closed convex subset of a Banach space E. Let $A$ be a weakly compact convex subset of $C$. Assume that $A$ has the fixed point property for nonexpansive mappings. Let $\left\{T_{n}: n \in \mathbb{N}\right\}$ be an infinite family of commuting nonexpansive mappings on $C$ such that

$$
T_{1}(A) \subset A, \quad T_{\ell+1}\left(A \cap\left(\bigcap_{k=1}^{\ell} F\left(T_{k}\right)\right)\right) \subset A
$$

for all $\ell \in \mathbb{N}$. Then there exists a common fixed point $z_{0} \in A$ of $\left\{T_{n}: n \in \mathbb{N}\right\}$.

Proof. We put $B_{\ell}:=A \cap\left(\bigcap_{k=1}^{\ell} F\left(T_{k}\right)\right)$ for $\ell \in \mathbb{N}$. We first show $B_{\ell}$ is nonempty and there exists a nonexpansive retraction $P_{\ell}$ from $A$ onto $B_{\ell}$ for all $\ell \in \mathbb{N}$. From the assumption of $T_{1}(A) \subset A$, there exists a fixed point $z_{1} \in A$ of $T_{1}$, that is, $B_{1} \neq \varnothing$. By Theorem 3.1, there exists a nonexpansive retraction $P_{1}$ from $A$ onto $B_{1}$. We assume $B_{\ell}$ is nonempty and there exists a nonexpansive retraction $P_{\ell}$ from $A$ onto $B_{\ell}$ for some $\ell \in \mathbb{N}$. From the assumption of $T_{\ell+1}\left(B_{\ell}\right) \subset A$, we have that $T_{\ell+1} \circ P_{\ell}$ is a nonexpansive mapping on $A$. We note that $B_{\ell+1}=F\left(T_{\ell+1} \circ P_{\ell}\right)$. Indeed, $B_{\ell+1} \subset F\left(T_{\ell+1} \circ P_{\ell}\right)$ is obvious. Conversely, we assume $z_{2} \in A$ satisfies $T_{\ell+1} \circ P_{\ell} z_{2}=z_{2}$. For $k \in \mathbb{N}$ with $k \leq \ell$, we have

$$
T_{k} z_{2}=T_{k} \circ T_{\ell+1} \circ P_{\ell} z_{2}=T_{\ell+1} \circ T_{k} \circ P_{\ell} z_{2}=T_{\ell+1} \circ P_{\ell} z_{2}=z_{2}
$$

that is, $z_{2} \in B_{\ell}$ and hence $P_{\ell} z_{2}=z_{2}$. Thus, we also have

$$
T_{\ell+1} z_{2}=T_{\ell+1} \circ P_{\ell} z_{2}=z_{2}
$$

Therefore $z_{2} \in B_{\ell+1}$ and hence $B_{\ell+1} \supset F\left(T_{\ell+1} \circ P_{\ell}\right)$. We have shown $B_{\ell+1}=F\left(T_{\ell+1} \circ P_{\ell}\right)$. Since $A$ has the fixed point property, we have

$$
B_{\ell+1}=F\left(T_{\ell+1} \circ P_{\ell}\right) \neq \varnothing .
$$

By Theorem 3.1 again, there exists a nonexpansive retraction $P_{\ell+1}$ from $A$ onto $B_{\ell+1}$. So, by induction, we have shown that $B_{\ell}$ is nonempty and there exists a nonexpansive retraction $P_{\ell}$ from $A$ onto $B_{\ell}$ for all $\ell \in \mathbb{N}$. Define a sequence $\left\{Q_{n}: n \in \mathbb{N}\right\}$ of nonexpansive mappings on $A$ by

$$
Q_{n}:=P_{n} \circ P_{n-1} \circ \cdots \circ P_{2} \circ P_{1}
$$

for $n \in \mathbb{N}$. Since $P_{m} x=P_{n} \circ P_{m} x$ for $x \in A, m, n \in \mathbb{N}$ with $m \geq n$, we have

$$
Q_{m} \circ Q_{n}=P_{\max \{m, n\}} \circ P_{\max \{m, n\}-1} \circ \cdots \circ P_{2} \circ P_{1}
$$

for all $m, n \in \mathbb{N}$ and hence $Q_{m} \circ Q_{n}=Q_{n} \circ Q_{m}$ for all $m, n \in \mathbb{N}$. So, by Theorem 3.1, there exists a common fixed point $z_{0} \in A$ of $\left\{Q_{n}: n \in \mathbb{N}\right\}$. Let us prove that $z_{0}$ is also a common fixed point of $\left\{T_{n}: n \in \mathbb{N}\right\}$. Since

$$
P_{1} z_{0}=Q_{1} z_{0}=z_{0},
$$


6 Infinite families of nonexpansive mappings

we have $z_{0} \in B_{1}$, that is, $T_{1} z_{0}=z_{0}$. We assume

$$
T_{1} z_{0}=T_{2} z_{0}=\cdots=T_{\ell} z_{0}=z_{0}
$$

for some $\ell \in \mathbb{N}$. Then

$$
\begin{aligned}
z_{0} & =Q_{\ell+1} z_{0}=P_{\ell+1} \circ P_{\ell} \circ \cdots \circ P_{2} \circ P_{1} z_{0} \\
& =P_{\ell+1} \circ P_{\ell} \circ \cdots \circ P_{2} z_{0}=\cdots=P_{\ell+1} \circ P_{\ell} z_{0}=P_{\ell+1} z_{0}
\end{aligned}
$$

and hence $z_{0} \in B_{\ell+1}$, that is, $T_{\ell+1} z_{0}=z_{0}$. So, by induction, $z_{0}$ is a common fixed point of $\left\{T_{n}: n \in \mathbb{N}\right\}$. This completes the proof.

\section{Lemmas}

In this section, we prove some lemmas which are used in the proofs of our main results.

Lemma 4.1. Let $C$ be a closed convex subset of a Banach space E. Let $\left\{T_{n}: n \in \mathbb{N}\right\}$ be an infinite family of commuting nonexpansive mappings on $C$ with a common fixed point. Let $\left\{\alpha_{n}\right\}$ and $\left\{t_{n}\right\}$ be sequences in $(0,1 / 2)$ satisfying $\lim _{n} t_{n}=\lim _{n} \alpha_{n} / t_{n}^{\ell}=0$ for $\ell \in \mathbb{N}$. Let $\left\{I_{n}\right\}$ be a sequence of nonempty subsets of $\mathbb{N}$ such that $I_{n} \subset I_{n+1}$ for $n \in \mathbb{N}$, and $\bigcup_{n=1}^{\infty} I_{n}=\mathbb{N}$. For $I \subset \mathbb{N}$ and $t \in(0,1 / 2)$ with $I \neq \varnothing$, define nonexpansive mappings $S(I, t)$ on $C$ by

$$
S(I, t) x:=\left(\left(1-\sum_{k \in I} t^{k}\right) T_{1} x+\sum_{k \in I} t^{k} T_{k+1} x\right)
$$

for $x \in C$. Fix $u \in C$ and define a sequence $\left\{u_{n}\right\}$ in $C$ by

$$
u_{n}=\left(1-\alpha_{n}\right) S\left(I_{n}, t_{n}\right) u_{n}+\alpha_{n} u
$$

for $n \in \mathbb{N}$. Let $\left\{u_{n_{\beta}}: \beta \in D\right\}$ be a subnet of $\left\{u_{n}\right\}$. Then the following hold.

(i) $\limsup \sup _{\beta}\left\|u_{n_{\beta}}-T_{1} x\right\| \leq \lim \sup _{\beta}\left\|u_{n_{\beta}}-x\right\|$ for $x \in C$.

(ii) If $x \in C$ satisfies $T_{1} x=x$, then $\limsup _{\beta}\left\|u_{n_{\beta}}-T_{2} x\right\| \leq \lim \sup _{\beta}\left\|u_{n_{\beta}}-x\right\|$.

(iii) If $x \in C$ satisfies $T_{1} x=T_{2} x=\cdots=T_{\ell-1} x=x$ for some $\ell \in \mathbb{N}$ with $\ell \geq 3$, then $\limsup \beta\left\|u_{n_{\beta}}-T_{\ell} x\right\| \leq \limsup \beta\left\|u_{n_{\beta}}-x\right\|$.

Proof. Let $v$ be a common fixed point of $\left\{T_{n}: n \in \mathbb{N}\right\}$. It is obvious that $S(I, t) v=v$ for all $I \subset \mathbb{N}$ and $t \in(0,1 / 2)$ with $I \neq \varnothing$. For $x \in C$ and $k \in \mathbb{N}$, we have

$$
\left\|T_{k} x\right\| \leq\left\|T_{k} x-v\right\|+\|v\|=\left\|T_{k} x-T_{k} v\right\|+\|v\| \leq\|x-v\|+\|v\| .
$$

Hence, $\left\{T_{k} x: k \in \mathbb{N}\right\}$ is bounded for every $x \in C$. Therefore $S(I, t)$ is well defined for every $I \subset \mathbb{N}$ and $t \in(0,1 / 2)$ with $I \neq \varnothing$. It is obvious that $S(I, t)$ is a nonexpansive mapping on $C$ for every $I$ and $t$. Since

$$
\begin{aligned}
\left\|u_{n}-v\right\| & =\left\|\left(1-\alpha_{n}\right) S\left(I_{n}, t_{n}\right) u_{n}+\alpha_{n} u-v\right\| \\
& \leq\left(1-\alpha_{n}\right)\left\|S\left(I_{n}, t_{n}\right) u_{n}-v\right\|+\alpha_{n}\|u-v\| \\
& \leq\left(1-\alpha_{n}\right)\left\|u_{n}-v\right\|+\alpha_{n}\|u-v\|,
\end{aligned}
$$


we have $\left\|u_{n}-v\right\| \leq\|u-v\|$ for $n \in \mathbb{N}$. Therefore $\left\{u_{n}\right\}$ is bounded. Since

$$
\left\|T_{k} u_{n}\right\| \leq\left\|T_{k} u_{n}-v\right\|+\|v\| \leq\left\|u_{n}-v\right\|+\|v\| \leq\left\|u_{n}\right\|+2\|v\|
$$

for all $n, k \in \mathbb{N},\left\{T_{k} u_{n}: n, k \in \mathbb{N}\right\}$ is also bounded. We fix $x \in C$ and we put

$$
M:=\max \left\{\|u\|,\|v\|, \sup _{n \in \mathbb{N}}\left\|u_{n}\right\|, \sup _{n, k \in \mathbb{N}}\left\|T_{k} u_{n}\right\|,\|x\|, \sup _{k \in \mathbb{N}}\left\|T_{k} x\right\|\right\}<\infty .
$$

It is obvious that $\left\|S(I, t) u_{n}\right\| \leq M$ and $\|S(I, t) x\| \leq M$ for all $n \in \mathbb{N}, I \subset \mathbb{N}$ and $t \in(0,1 / 2)$ with $I \neq \varnothing$. From the assumption, we have

$$
S\left(I_{n}, t_{n}\right) u_{n}-u_{n}=\alpha_{n}\left(S\left(I_{n}, t_{n}\right) u_{n}-u\right)
$$

for $n \in \mathbb{N}$. We have

$$
\begin{aligned}
\left\|u_{n_{\beta}}-T_{1} x\right\| & \leq\left\|u_{n_{\beta}}-S\left(I_{n_{\beta}}, t_{n_{\beta}}\right) u_{n_{\beta}}\right\|+\left\|S\left(I_{n_{\beta}}, t_{n_{\beta}}\right) u_{n_{\beta}}-S\left(I_{n_{\beta}}, t_{n_{\beta}}\right) x\right\|+\left\|S\left(I_{n_{\beta}}, t_{n_{\beta}}\right) x-T_{1} x\right\| \\
& \leq \alpha_{n_{\beta}}\left\|S\left(I_{n_{\beta}}, t_{n_{\beta}}\right) u_{n_{\beta}}-u\right\|+\left\|u_{n_{\beta}}-x\right\|+\left\|-\sum_{k \in I_{n_{\beta}}} t_{n_{\beta}}^{k} T_{1} x+\sum_{k \in I_{n_{\beta}}} t_{n_{\beta}}^{k} T_{k+1} x\right\| \\
& \leq 2 M \alpha_{n_{\beta}}+\left\|u_{n_{\beta}}-x\right\|+2 M \sum_{k \in I_{n_{\beta}}} t_{n_{\beta}}^{k} \leq 2 M \alpha_{n_{\beta}}+\left\|u_{n_{\beta}}-x\right\|+2 M \frac{t_{n_{\beta}}}{1-t_{n_{\beta}}}
\end{aligned}
$$

for $\beta \in D$ and hence

$$
\limsup _{\beta \in D}\left\|u_{n_{\beta}}-T_{1} x\right\| \leq \underset{\beta \in D}{\limsup }\left\|u_{n_{\beta}}-x\right\| .
$$

This is (i). We next show (ii). We assume that $T_{1} x=x$. Then $T_{1} \circ T_{2} x=T_{2} \circ T_{1} x=T_{2} x$. For $\beta \in D$ with $1,2 \in I_{n_{\beta}}$, we have

$$
\begin{aligned}
\left\|u_{n_{\beta}}-T_{2} x\right\| \leq & \left\|u_{n_{\beta}}-S\left(I_{n_{\beta}}, t_{n_{\beta}}\right) u_{n_{\beta}}\right\|+\left\|S\left(I_{n_{\beta}}, t_{n_{\beta}}\right) u_{n_{\beta}}-T_{2} x\right\| \\
\leq & \alpha_{n_{\beta}}\left\|S\left(I_{n_{\beta}}, t_{n_{\beta}}\right) u_{n_{\beta}}-u\right\|+\left(1-\sum_{k \in I_{n_{\beta}}} t_{n_{\beta}}^{k}\right)\left\|T_{1} u_{n_{\beta}}-T_{2} x\right\| \\
& +t_{n_{\beta}}\left\|T_{2} u_{n_{\beta}}-T_{2} x\right\|+\sum_{k \in I_{n_{\beta}} \backslash\{1\}} t_{n_{\beta}}^{k}\left\|T_{k+1} u_{n_{\beta}}-T_{2} x\right\| \\
\leq & 2 M \alpha_{n_{\beta}}+\left(1-t_{n_{\beta}}\right)\left\|T_{1} u_{n_{\beta}}-T_{2} x\right\|+t_{n_{\beta}}\left\|u_{n_{\beta}}-x\right\|+2 M \sum_{k \in I_{n_{\beta}} \backslash\{1\}} t_{n_{\beta}}^{k} \\
\leq & 2 M \alpha_{n_{\beta}}+\left(1-t_{n_{\beta}}\right)\left\|T_{1} u_{n_{\beta}}-T_{1} \circ T_{2} x\right\|+t_{n_{\beta}}\left\|u_{n_{\beta}}-x\right\|+2 M \frac{t_{n_{\beta}}^{2}}{1-t_{n_{\beta}}} \\
\leq & 2 M \alpha_{n_{\beta}}+\left(1-t_{n_{\beta}}\right)\left\|u_{n_{\beta}}-T_{2} x\right\|+t_{n_{\beta}}\left\|u_{n_{\beta}}-x\right\|+2 M \frac{t_{n_{\beta}}^{2}}{1-t_{n_{\beta}}}
\end{aligned}
$$


8 Infinite families of nonexpansive mappings

and hence

$$
\left\|u_{n_{\beta}}-T_{2} x\right\| \leq 2 M \frac{\alpha_{n_{\beta}}}{t_{n_{\beta}}}+\left\|u_{n_{\beta}}-x\right\|+2 M \frac{t_{n_{\beta}}}{1-t_{n_{\beta}}} .
$$

Therefore we obtain

$$
\limsup _{\beta \in D}\left\|u_{n_{\beta}}-T_{2} x\right\| \leq \limsup _{\beta \in D}\left\|u_{n_{\beta}}-x\right\|
$$

Let us prove (iii). We assume $T_{1} x=T_{2} x=\cdots=T_{\ell-1} x=x$ for some $\ell \in \mathbb{N}$ with $\ell \geq$ 3. Then $T_{m} \circ T_{\ell} x=T_{\ell} \circ T_{m} x=T_{\ell} x$ for every $m \in \mathbb{N}$ with $1 \leq m<\ell$. For $\beta \in D$ with $1,2, \ldots, \ell-1 \in I_{n_{\beta}}$, we have

$$
\begin{aligned}
& \left\|u_{n_{\beta}}-T_{\ell} x\right\| \leq\left\|u_{n_{\beta}}-S\left(I_{n_{\beta}}, t_{n_{\beta}}\right) u_{n_{\beta}}\right\|+\left\|S\left(I_{n_{\beta}}, t_{n_{\beta}}\right) u_{n_{\beta}}-T_{\ell} x\right\| \\
& \leq \alpha_{n_{\beta}}\left\|S\left(I_{n_{\beta}}, t_{n_{\beta}}\right) u_{n_{\beta}}-u\right\|+\left(1-\sum_{k \in I_{n_{\beta}}} t_{n_{\beta}}^{k}\right)\left\|T_{1} u_{n_{\beta}}-T_{\ell} x\right\| \\
& +\sum_{m=1}^{\ell-2} t_{n_{\beta}}^{m}\left\|T_{m+1} u_{n_{\beta}}-T_{\ell} x\right\|+t_{n_{\beta}}^{\ell-1}\left\|T_{\ell} u_{n_{\beta}}-T_{\ell} x\right\| \\
& +\sum_{k \in I_{n_{\beta}} \backslash\{1,2, \ldots, \ell-1\}} t_{n_{\beta}}^{k}\left\|T_{k+1} u_{n_{\beta}}-T_{\ell} x\right\| \\
& \leq 2 M \alpha_{n_{\beta}}+\left(1-\sum_{m=1}^{\ell-1} t_{n_{\beta}}^{m}\right)\left\|T_{1} u_{n_{\beta}}-T_{\ell} x\right\| \\
& +\sum_{m=1}^{\ell-2} t_{n_{\beta}}^{m}\left\|T_{m+1} u_{n_{\beta}}-T_{\ell} x\right\|+t_{n_{\beta}}^{\ell-1}\left\|u_{n_{\beta}}-x\right\|+2 M \sum_{k \in I_{n_{\beta}} \backslash\{1,2, \ldots, \ell-1\}} t_{n_{\beta}}^{k} \\
& \leq 2 M \alpha_{n_{\beta}}+\left(1-\sum_{m=1}^{\ell-1} t_{n_{\beta}}^{m}\right)\left\|T_{1} u_{n_{\beta}}-T_{1} \circ T_{\ell} x\right\| \\
& +\sum_{m=1}^{\ell-2} t_{n_{\beta}}^{m}\left\|T_{m+1} u_{n_{\beta}}-T_{m+1} \circ T_{\ell} x\right\|+t_{n_{\beta}}^{\ell-1}\left\|u_{n_{\beta}}-x\right\|+2 M \frac{t_{n_{\beta}}^{\ell}}{1-t_{n_{\beta}}} \\
& \leq 2 M \alpha_{n_{\beta}}+\left(1-\sum_{m=1}^{\ell-1} t_{n_{\beta}}^{m}\right)\left\|u_{n_{\beta}}-T_{\ell} x\right\| \\
& +\sum_{m=1}^{\ell-2} t_{n_{\beta}}^{m}\left\|u_{n_{\beta}}-T_{\ell} x\right\|+t_{n_{\beta}}^{\ell-1}\left\|u_{n_{\beta}}-x\right\|+2 M \frac{t_{n_{\beta}}^{\ell}}{1-t_{n_{\beta}}} \\
& =2 M \alpha_{n_{\beta}}+\left(1-t_{n_{\beta}}^{\ell-1}\right)\left\|u_{n_{\beta}}-T_{\ell} x\right\|+t_{n_{\beta}}^{\ell-1}\left\|u_{n_{\beta}}-x\right\|+2 M \frac{t_{n_{\beta}}^{\ell}}{1-t_{n_{\beta}}}
\end{aligned}
$$


and hence

$$
\left\|u_{n_{\beta}}-T_{\ell} x\right\| \leq 2 M \frac{\alpha_{n_{\beta}}}{t_{n_{\beta}}^{\ell-1}}+\left\|u_{n_{\beta}}-x\right\|+2 M \frac{t_{n_{\beta}}}{1-t_{n_{\beta}}} .
$$

Therefore we obtain

$$
\limsup _{\beta \in D}\left\|u_{n_{\beta}}-T_{\ell} x\right\| \leq \underset{\beta \in D}{\limsup }\left\|u_{n_{\beta}}-x\right\| .
$$

This completes the proof.

Remark 4.2. Let $g$ be a strictly increasing mapping on $\mathbb{N}$. Then it is obvious that $\lim _{n} t_{g(n)}$ $=\lim _{n} \alpha_{g(n)} / t_{g(n)}^{\ell}=0$ for all $\ell \in \mathbb{N}, I_{g(n)} \subset I_{g(n+1)}$ for $n \in \mathbb{N}$, and $\bigcup_{n=1}^{\infty} I_{g(n)}=\mathbb{N}$. Thus, the same conclusions of Lemmas 4.3-4.6 also hold for $\left\{u_{g(n)}\right\}$.

Lemma 4.3. Let $E, C,\left\{T_{n}\right\},\left\{\alpha_{n}\right\},\left\{t_{n}\right\},\left\{I_{n}\right\}, u$, and $\left\{u_{n}\right\}$ be as in Lemma 4.1. Assume that $\left\{u_{n}\right\}$ converges strongly to some point $x \in C$. Then $x$ is a common fixed point of $\left\{T_{n}: n \in \mathbb{N}\right\}$.

Proof. From Lemma 4.1(i), we have

$$
\limsup _{n \rightarrow \infty}\left\|u_{n}-T_{1} x\right\| \leq \lim _{n \rightarrow \infty}\left\|u_{n}-x\right\|=0
$$

This means $\left\{u_{n}\right\}$ converges to $T_{1} x$ and hence $T_{1} x=x$. We assume that $T_{1} x=\cdots=$ $T_{\ell-1} x=x$ for some $\ell \in \mathbb{N}$ with $\ell \geq 2$. Then from Lemma 4.1(ii) and (iii), we have

$$
\limsup _{n \rightarrow \infty}\left\|u_{n}-T_{\ell} x\right\| \leq \lim _{n \rightarrow \infty}\left\|u_{n}-x\right\|=0
$$

This means $\left\{u_{n}\right\}$ converges to $T_{\ell} x$ and hence $T_{\ell} x=x$. So, by induction, we obtain $T_{n} x=x$ for all $n \in \mathbb{N}$. This completes the proof.

Lemma 4.4. Let $E, C,\left\{T_{n}\right\},\left\{\alpha_{n}\right\},\left\{t_{n}\right\},\left\{I_{n}\right\}, u$, and $\left\{u_{n}\right\}$ be as in Lemma 4.1. Assume that $E$ is smooth and $z \in C$ is a common fixed point of $\left\{T_{n}: n \in \mathbb{N}\right\}$. Then

$$
\left\langle u_{n}-u, J\left(u_{n}-z\right)\right\rangle \leq 0
$$

for all $n \in \mathbb{N}$.

Proof. Since $\alpha_{n}\left(u_{n}-u\right)=\left(1-\alpha_{n}\right)\left(S\left(I_{n}, t_{n}\right) u_{n}-u_{n}\right)$, we have

$$
\begin{aligned}
\frac{\alpha_{n}}{1-\alpha_{n}}\left\langle u_{n}-u, J\left(u_{n}-z\right)\right\rangle & =\left\langle S\left(I_{n}, t_{n}\right) u_{n}-u_{n}, J\left(u_{n}-z\right)\right\rangle \\
& =\left\langle S\left(I_{n}, t_{n}\right) u_{n}-z, J\left(u_{n}-z\right)\right\rangle+\left\langle z-u_{n}, J\left(u_{n}-z\right)\right\rangle \\
& =\left\langle S\left(I_{n}, t_{n}\right) u_{n}-S\left(I_{n}, t_{n}\right) z, J\left(u_{n}-z\right)\right\rangle-\left\|u_{n}-z\right\|^{2} \\
& \leq\left\|S\left(I_{n}, t_{n}\right) u_{n}-S\left(I_{n}, t_{n}\right) z\right\|\left\|u_{n}-z\right\|-\left\|u_{n}-z\right\|^{2} \\
& \leq\left\|u_{n}-z\right\|^{2}-\left\|u_{n}-z\right\|^{2}=0 .
\end{aligned}
$$


Thus we obtain

$$
\left\langle u_{n}-u, J\left(u_{n}-z\right)\right\rangle \leq 0
$$

for all $n \in \mathbb{N}$.

Lemma 4.5. Let $E, C,\left\{T_{n}\right\},\left\{\alpha_{n}\right\},\left\{t_{n}\right\},\left\{I_{n}\right\}, u$, and $\left\{u_{n}\right\}$ be as in Lemma 4.1. Assume that $E$ is smooth. Then $\left\{u_{n}\right\}$ has at most one cluster point.

Proof. We assume that a subsequence $\left\{u_{n_{i}}\right\}$ of $\left\{u_{n}\right\}$ converges strongly to $x$, and that another subsequence $\left\{u_{n_{j}}\right\}$ of $\left\{u_{n}\right\}$ converges strongly to $y$. Applying Lemma 4.3 to the subsequences $\left\{u_{n_{i}}\right\}$ and $\left\{u_{n_{j}}\right\}$, we have that $x$ and $y$ are common fixed points of $\left\{T_{n}: n \in\right.$ $\mathbb{N}\}$. So, by Lemma 4.4, we have

$$
\left\langle u_{n_{i}}-u, J\left(u_{n_{i}}-y\right)\right\rangle \leq 0
$$

for all $i \in \mathbb{N}$. Therefore we obtain

$$
\langle x-u, J(x-y)\rangle \leq 0 .
$$

Similarly we can prove

$$
\langle y-u, J(y-x)\rangle \leq 0 \text {. }
$$

So we obtain

$$
\begin{aligned}
\|x-y\|^{2} & =\langle x-y, J(x-y)\rangle \\
& =\langle x-u, J(x-y)\rangle+\langle u-y, J(x-y)\rangle \\
& =\langle x-u, J(x-y)\rangle+\langle y-u, J(y-x)\rangle \leq 0 .
\end{aligned}
$$

This implies $x=y$. This completes the proof.

Lemma 4.6. Let E be a reflexive Banach space with uniformly Gâteaux differentiable norm and let $C$ be a closed convex subset of $E$ with the fixed point property for nonexpansive mappings. Let $\left\{T_{n}\right\},\left\{\alpha_{n}\right\},\left\{t_{n}\right\},\left\{I_{n}\right\}, u$, and $\left\{u_{n}\right\}$ be as in Lemma 4.1. Then $\left\{u_{n}\right\}$ has a cluster point which is a common fixed point of $\left\{T_{n}: n \in \mathbb{N}\right\}$.

Proof. From the proof of Lemma 4.1, we have that $\left\{u_{n}\right\}$ is bounded. Take a universal subnet $\left\{u_{v}: \nu \in D\right\}$ of $\left\{u_{n}\right\}$. Define a continuous convex function $f$ from $C$ into $[0, \infty)$ by

$$
f(x):=\lim _{v \in D}\left\|u_{v}-x\right\|
$$

for all $x \in C$. We note that $f$ is well defined because $\left\{\left\|u_{v}-x\right\|\right\}$ is a universal net in some compact subset of $\mathbb{R}$ for each $x \in C$. From the reflexivity of $E$ and $\lim _{\|x\| \rightarrow \infty} f(x)=\infty$, we can put $r:=\min _{x \in C} f(x)$ and define a nonempty weakly compact convex subset $A$ of $C$ by

$$
A:=\{x \in C: f(x)=r\} .
$$


We will prove that $A$ satisfies the assumption of Theorem 3.2. For each $x \in A$, by Lemma 4.1(i), we have

$$
r \leq f\left(T_{1} x\right)=\lim _{v \in D}\left\|u_{v}-T_{1} x\right\| \leq \lim _{v \in D}\left\|u_{v}-x\right\|=f(x)=r
$$

and hence $T_{1} x \in A$. This implies $A$ is $T_{1}$-invariant. Fix $x \in A$ with $T_{1} x=\cdots=T_{\ell} x$ for some $\ell \in \mathbb{N}$. Then by Lemma 4.1(ii) and (iii), we have

$$
r \leq f\left(T_{\ell+1} x\right)=\lim _{v \in D}\left\|u_{v}-T_{\ell+1} x\right\| \leq \lim _{\nu \in D}\left\|u_{v}-x\right\|=f(x)=r
$$

and hence $T_{\ell+1} x \in A$. Thus we obtain $T_{\ell+1}\left(A \cap\left(\bigcap_{k=1}^{\ell} F\left(T_{k}\right)\right)\right) \subset A$ for all $\ell \in \mathbb{N}$. So, by Theorem 3.2, there exists a common fixed point $z$ of $\left\{T_{n}: n \in \mathbb{N}\right\}$ in $A$. We next prove that such $z$ is a cluster point of $\left\{u_{n}\right\}$. By Lemma 4.4 , we have

$$
\left\langle u_{v}-u, J\left(u_{v}-z\right)\right\rangle \leq 0
$$

for all $v \in D$. On the other hand, from $z \in A$, we have

$$
\lim _{v \in D}\left\langle u-z, J\left(u_{v}-z\right)\right\rangle \leq 0
$$

by Lemma 2.2. Hence,

$$
\begin{aligned}
\lim _{v \in D}\left\|u_{v}-z\right\|^{2} & =\lim _{v \in D}\left\langle u_{v}-z, J\left(u_{v}-z\right)\right\rangle \\
& =\lim _{v \in D}\left\langle u_{v}-u, J\left(u_{v}-z\right)\right\rangle+\lim _{v \in D}\left\langle u-z, J\left(u_{v}-z\right)\right\rangle \leq 0
\end{aligned}
$$

holds. Therefore

$$
\liminf _{n \rightarrow \infty}\left\|u_{n}-z\right\| \leq \lim _{v \in D}\left\|u_{v}-z\right\|=0
$$

that is, $z$ is a cluster point of $\left\{u_{n}\right\}$. This completes the proof.

Lemma 4.7. Let $E, C,\left\{T_{n}\right\},\left\{\alpha_{n}\right\},\left\{t_{n}\right\},\left\{I_{n}\right\}, u$, and $\left\{u_{n}\right\}$ be as in Lemma 4.1. Assume that $E$ is smooth. For each $u \in C$, define a sequence $\{Q(u, n)\}$ in $C$ by

$$
Q(u, n)=\left(1-\alpha_{n}\right) S\left(I_{n}, t_{n}\right) Q(u, n)+\alpha_{n} u
$$

for $n \in \mathbb{N}$. Suppose that for every $u \in C,\{Q(u, n)\}$ converges strongly. Then

$$
P u=\lim _{n \rightarrow \infty} Q(u, n)
$$

holds for every $u \in C$, where $P$ is the unique sunny nonexpansive retraction from $C$ onto $\bigcap_{n=1}^{\infty} F\left(T_{n}\right)$.

Proof. We put $F(\mathscr{S}):=\bigcap_{n=1}^{\infty} F\left(T_{n}\right)$. Define a mapping $P$ on $C$ by $P u:=\lim _{n} Q(u, n)$ for $u \in C$. We will prove that such $P$ is the unique sunny nonexpansive retraction from $C$ onto $F(\mathscr{S})$. By Lemma 4.3, we note that $P x \in F(\mathscr{Y})$ for all $x \in C$. For $z \in F(\mathscr{Y})$, since

$$
z=\left(1-\alpha_{n}\right) S\left(I_{n}, t_{n}\right) z+\alpha_{n} z
$$


for all $n \in \mathbb{N}$, we have $Q(z, n)=z$ for all $n \in \mathbb{N}$. Hence, we obtain $P z=z$. Therefore we have shown that $P^{2}=P$, that is, $P$ is a retraction from $C$ onto $F(\mathscr{Y})$. Fix $x \in C$ and $y \in$ $F(\mathscr{S})$. Then, from Lemma 4.4 , we have

$$
\langle Q(x, n)-x, J(Q(x, n)-y)\rangle \leq 0
$$

for all $n \in \mathbb{N}$. Since $\{Q(x, n)\}$ converges strongly to $P x$, we obtain

$$
\langle P x-x, J(P x-y)\rangle \leq 0 .
$$

So, by Lemma 2.1, such mapping $P$ is the unique sunny nonexpansive retraction from $C$ onto $F(\mathscr{Y})$. This completes the proof.

\section{Main results}

In this section, we prove our main results. We put $F(\mathscr{S}):=\bigcap_{n=1}^{\infty} F\left(T_{n}\right)$.

Theorem 5.1. Let E be a reflexive Banach space with uniformly Gâteaux differentiable norm and let $C$ be a closed convex subset of $E$ with the fixed point property for nonexpansive mappings. Let $\left\{T_{n}\right\},\left\{\alpha_{n}\right\},\left\{t_{n}\right\},\left\{I_{n}\right\}, u$, and $\left\{u_{n}\right\}$ be as in Lemma 4.1. Then $\left\{u_{n}\right\}$ converges strongly to $P u$, where $P$ is the unique sunny nonexpansive retraction from $C$ onto $F(\mathscr{S})$.

Proof. Applying Lemma 4.6 to a subsequence of $\left\{u_{n}\right\}$, we have that every subsequence of $\left\{u_{n}\right\}$ has a cluster point. So, by Lemmas 2.4 and 4.5 , we obtain that $\left\{u_{n}\right\}$ converges strongly. So, by Lemma 4.7, we obtain the desired result.

THeOREM 5.2. Let $E$ be a smooth reflexive Banach space with the Opial property and let $C$ be a closed convex subset of E. Assume that the duality mapping J of $E$ is weakly sequentially continuous at zero. Let $\left\{T_{n}\right\},\left\{\alpha_{n}\right\},\left\{t_{n}\right\},\left\{I_{n}\right\}, u$, and $\left\{u_{n}\right\}$ be as in Lemma 4.1. Then $\left\{u_{n}\right\}$ converges strongly to $P u$, where $P$ is the unique sunny nonexpansive retraction from $C$ onto $F(\mathscr{Y})$.

Proof. From the proof of Lemma 4.1, we have that $\left\{u_{n}\right\}$ is bounded. Let $\left\{u_{n_{i}}\right\}$ be an arbitrary subsequence of $\left\{u_{n}\right\}$. Since $E$ is reflexive, there exists a subsequence $\left\{u_{n_{i_{j}}}\right\}$ of $\left\{u_{n_{i}}\right\}$ which converges weakly to some point $z \in C$. We put $z_{j}:=u_{n_{i_{j}}}$ for $j \in \mathbb{N}$. Applying Lemma $4.1(\mathrm{i})$ to $\left\{z_{j}\right\}$, we have

$$
\limsup _{j \rightarrow \infty}\left\|z_{j}-T_{1} z\right\| \leq \underset{j \rightarrow \infty}{\limsup }\left\|z_{j}-z\right\| .
$$

Since $E$ has the Opial property, we obtain $T_{1} z=z$. We assume that $T_{1} z=\cdots=T_{\ell} z=z$ for some $\ell \in \mathbb{N}$. Then, by Lemma 4.1(ii) and (iii), we have

$$
\limsup _{j \rightarrow \infty}\left\|z_{j}-T_{\ell+1} z\right\| \leq \underset{j \rightarrow \infty}{\limsup }\left\|z_{j}-z\right\|
$$


By the Opial property of $E$ again, we obtain $T_{\ell+1} z=z$. Thus, by induction, $z$ is a common fixed point of $\left\{T_{n}: n \in \mathbb{N}\right\}$. By using Lemma 4.4 , we have

$$
\begin{aligned}
\left\|z_{j}-z\right\|^{2} & =\left\langle z_{j}-z, J\left(z_{j}-z\right)\right\rangle \\
& =\left\langle z_{j}-u, J\left(z_{j}-z\right)\right\rangle+\left\langle u-z, J\left(z_{j}-z\right)\right\rangle \\
& \leq\left\langle u-z, J\left(z_{j}-z\right)\right\rangle
\end{aligned}
$$

for all $j \in \mathbb{N}$. Since $J$ is weakly sequentially continuous at zero, $\left\{z_{j}\right\}$ converges strongly to $z$. Hence, $\left\{u_{n_{i}}\right\}$ has a cluster point $z$. So, by Lemmas 2.4 and $4.5,\left\{u_{n}\right\}$ itself converges strongly. Thus, by Lemma 4.7, we obtain the desired result.

Remark 5.3. In Theorems 5.1 and 5.2, from the proofs of Lemma 4.6 and Theorem 5.2, we may replace the condition of the reflexivity of $E$ by the weaker condition that $C$ is locally weakly compact.

By Theorems 5.1 and 5.2, we obtain the following.

Theorem 5.4. Let $C$ be a weakly compact convex subset of a Banach space E. Assume that either of the following holds:

(i) E is uniformly smooth; or

(ii) $E$ is a smooth Banach space with the Opial property and the duality mapping $J$ of $E$ is weakly sequentially continuous at zero.

Let $\left\{T_{n}: n \in \mathbb{N}\right\}$ be an infinite family of commuting nonexpansive mappings on C. Let $\left\{\alpha_{n}\right\}$ and $\left\{t_{n}\right\}$ be sequences in $(0,1 / 2)$ satisfying $\lim _{n} t_{n}=\lim _{n} \alpha_{n} / t_{n}^{\ell}=0$ for $\ell \in \mathbb{N}$. Let $\left\{I_{n}\right\}$ be a sequence of nonempty subsets of $\mathbb{N}$ such that $I_{n} \subset I_{n+1}$ for $n \in \mathbb{N}$, and $\bigcup_{n=1}^{\infty} I_{n}=\mathbb{N}$. Fix $u \in C$ and define a sequence $\left\{u_{n}\right\}$ in $C$ by

$$
u_{n}=\left(1-\alpha_{n}\right)\left(\left(1-\sum_{k \in I_{n}} t_{n}^{k}\right) T_{1} u_{n}+\sum_{k \in I_{n}} t_{n}^{k} T_{k+1} u_{n}\right)+\alpha_{n} u
$$

for $n \in \mathbb{N}$. Then $\left\{u_{n}\right\}$ converges strongly to $P u$, where $P$ is the unique sunny nonexpansive retraction from $C$ onto $F(\mathscr{S})$.

Remark 5.5. By Theorem 3.1, we know $F(\mathscr{S}) \neq \varnothing$.

Example 5.6. Define sequences $\left\{\alpha_{n}\right\}$ and $\left\{t_{n}\right\}$ by $\alpha_{n}:=1 / n^{n}$ and $t_{n}:=1 / n$ for $n \in \mathbb{N}$. Then $\left\{\alpha_{n}\right\}$ and $\left\{t_{n}\right\}$ satisfy $\lim _{n} t_{n}=\lim _{n} \alpha_{n} / t_{n}^{\ell}=0$ for $\ell \in \mathbb{N}$.

Corollary 5.7. Let $E, C,\left\{T_{n}\right\},\left\{\alpha_{n}\right\},\left\{t_{n}\right\}$, and $P$ be as in Theorem 5.4. Fix $u \in C$ and define sequences $\left\{u_{n}\right\}$ and $\left\{v_{n}\right\}$ in $C$ by

$$
\begin{aligned}
& u_{n}=\left(1-\alpha_{n}\right)\left(\left(1-\sum_{k=1}^{n} t_{n}^{k}\right) T_{1} u_{n}+\sum_{k=1}^{n} t_{n}^{k} T_{k+1} u_{n}\right)+\alpha_{n} u, \\
& v_{n}=\left(1-\alpha_{n}\right)\left(\left(1-\sum_{k=1}^{\infty} t_{n}^{k}\right) T_{1} v_{n}+\sum_{k=1}^{\infty} t_{n}^{k} T_{k+1} v_{n}\right)+\alpha_{n} u
\end{aligned}
$$

for $n \in \mathbb{N}$. Then $\left\{u_{n}\right\}$ and $\left\{v_{n}\right\}$ converge strongly to $P u$. 
From the proofs of lemmas in Section 4, we also obtain the following.

Theorem 5.8. Let $E$ and $C$ be as in Theorem 5.4. Let $\left\{T_{n}: n=1,2, \ldots, \ell\right\}$ be a finite family of commuting nonexpansive mappings on $C$. Let $\left\{\alpha_{n}\right\}$ and $\left\{t_{n}\right\}$ be sequences in $(0,1 / 2)$ satisfying $\lim _{n} t_{n}=\lim _{n} \alpha_{n} / t_{n}^{\ell-1}=0$. Fix $u \in C$ and define a sequence $\left\{u_{n}\right\}$ in $C$ by

$$
u_{n}=\left(1-\alpha_{n}\right)\left(\left(1-\sum_{k=1}^{\ell-1} t_{n}^{k}\right) T_{1} u_{n}+\sum_{k=1}^{\ell-1} t_{n}^{k} T_{k+1} u_{n}\right)+\alpha_{n} u
$$

for $n \in \mathbb{N}$. Then $\left\{u_{n}\right\}$ converges strongly to $P u$, where $P$ is the unique sunny nonexpansive retraction from $C$ onto $\bigcap_{k=1}^{\ell} F\left(T_{k}\right)$.

\section{6. $\ell$-parameter nonexpansive semigroups}

In this section, we apply Theorem 5.8 to $\ell$-parameter nonexpansive semigroups. We recall that a family of mappings $\left\{T(p): p \in[0, \infty)^{\ell}\right\}$ is said to be an $\ell$-parameter nonexpansive semigroup on a closed convex subset $C$ of a Banach space $E$ if the following are satisfied.

(i) For each $p \in[0, \infty)^{\ell}, T(p)$ is a nonexpansive mapping on $C$.

(ii) $T(p+q)=T(p) \circ T(q)$ for all $p, q \in[0, \infty)^{\ell}$.

(iii) For each $x \in C$, the mapping $p \mapsto T(p) x$ from $[0, \infty)^{\ell}$ into $C$ is continuous.

The following is proved in [22]. See also [23].

Theorem 6.1 [22]. Let $\left\{T(p): p \in[0, \infty)^{\ell}\right\}$ be an $\ell$-parameter nonexpansive semigroup on a closed convex subset $C$ of a Banach space E. Let $p_{1}, p_{2}, \ldots, p_{\ell} \in[0, \infty)^{\ell}$ such that $\left\{p_{1}, p_{2}, \ldots, p_{\ell}\right\}$ is linearly independent in the usual sense. Let $\beta_{1}, \beta_{2}, \ldots, \beta_{\ell} \in \mathbb{R}$ such that $\left\{1, \beta_{1}, \beta_{2}, \ldots, \beta_{\ell}\right\}$ is linearly independent over $\mathbb{Q}$, that is,

$$
\nu_{0}+\nu_{1} \beta_{1}+\nu_{2} \beta_{2}+\cdots+v_{\ell} \beta_{\ell}=0 \quad \text { implies } \nu_{0}=\nu_{1}=\nu_{2}=\cdots=v_{\ell}=0
$$

for $\nu_{0}, v_{1}, \nu_{2}, \ldots, v_{\ell} \in \mathbb{Z}$. Suppose $p_{0}:=\beta_{1} p_{1}+\beta_{2} p_{2}+\cdots+\beta_{\ell} p_{\ell} \in[0, \infty)^{\ell}$. Then

$$
\bigcap_{p \in[0, \infty)^{\ell}} F(T(p))=F\left(T\left(p_{0}\right)\right) \cap F\left(T\left(p_{1}\right)\right) \cap F\left(T\left(p_{2}\right)\right) \cap \cdots \cap F\left(T\left(p_{\ell}\right)\right)
$$

holds.

By Theorems 5.8 and 6.1, we obtain the following.

Theorem 6.2. Let $E$ and $C$ be as in Theorem 5.4. Let $\{T(p)\},\left\{p_{0}, p_{1}, p_{2}, \ldots, p_{\ell}\right\},\left\{\beta_{1}, \beta_{2}, \ldots\right.$, $\left.\beta_{\ell}\right\}$ be as in Theorem 6.1. Let $\left\{\alpha_{n}\right\}$ and $\left\{t_{n}\right\}$ be sequences in $(0,1 / 2)$ satisfying $\lim _{n} t_{n}=$ $\lim _{n} \alpha_{n} / t_{n}^{\ell}=0$. Fix $u \in C$ and define a sequence $\left\{u_{n}\right\}$ in $C$ by

$$
u_{n}=\left(1-\alpha_{n}\right)\left(\left(1-\sum_{k=1}^{\ell} t_{n}^{k}\right) T\left(p_{0}\right) u_{n}+\sum_{k=1}^{\ell} t_{n}^{k} T\left(p_{k}\right) u_{n}\right)+\alpha_{n} u
$$

for $n \in \mathbb{N}$. Then $\left\{u_{n}\right\}$ converges strongly to $P u$, where $P$ is the unique sunny nonexpansive retraction from $C$ onto $\bigcap_{p \in[0, \infty)^{\ell}} F(T(p))$. 
When $\ell=1$, Theorem 6.2 becomes the following, which differs from Theorem 1.2.

Corollary 6.3. Let $E$ and $C$ be as in Theorem 5.4. Let $\{T(t): t \geq 0\}$ be a one-parameter nonexpansive semigroup on C. Let $\left\{\alpha_{n}\right\}$ and $\left\{t_{n}\right\}$ be sequences in $(0,1 / 2)$ satisfying $\lim _{n} t_{n}=$ $\lim _{n} \alpha_{n} / t_{n}=0$. Let $\sigma$ and $\tau$ be positive real numbers satisfying $\sigma / \tau \notin \mathbb{Q}$. Fix $u \in C$ and define sequences $\left\{u_{n}\right\}$ and $\left\{v_{n}\right\}$ in $C$ by

$$
\begin{aligned}
& u_{n}=\left(1-\alpha_{n}\right)\left(\left(1-t_{n}\right) T(\sigma) u_{n}+t_{n} T(\tau) u_{n}\right)+\alpha_{n} u, \\
& v_{n}=\left(1-t_{n}-\alpha_{n}\right) T(\sigma) v_{n}+t_{n} T(\tau) v_{n}+\alpha_{n} u
\end{aligned}
$$

for $n \in \mathbb{N}$. Then $\left\{u_{n}\right\}$ and $\left\{v_{n}\right\}$ converge strongly to $P u$, where $P$ is the unique sunny nonexpansive retraction from $C$ onto $\bigcap_{t \geq 0} F(T(t))$.

Proof. We remark that

$$
\begin{gathered}
v_{n}=\left(1-\alpha_{n}\right)\left(\left(1-\frac{t_{n}}{1-\alpha_{n}}\right) T(\sigma) v_{n}+\frac{t_{n}}{1-\alpha_{n}} T(\tau) v_{n}\right)+\alpha_{n} u, \\
\lim _{n \rightarrow \infty} \frac{\alpha_{n}}{t_{n} /\left(1-\alpha_{n}\right)}=\lim _{n \rightarrow \infty} \frac{\alpha_{n}\left(1-\alpha_{n}\right)}{t_{n}}=0 .
\end{gathered}
$$

From this thing, we can obtain the desired result.

\section{Acknowledgment}

The author is supported in part by Grants-in-Aid for Scientific Research from the Japanese Ministry of Education, Culture, Sports, Science, and Technology.

\section{References}

[1] J.-B. Baillon, Quelques aspects de la théorie des points fixes dans les espaces de Banach. I, II, Séminaire d'Analyse Fonctionnelle (1978-1979) Exp. No. 7-8, École Polytech, Palaiseau, 1979, p. 45 .

[2] S. Banach, Sur les opérations dans les ensembles abstraits et leur application aux équations intégrales, Fundamenta Mathematicae 3 (1922), 133-181.

[3] M. S. Brodskii and D. P. Mil'man, On the center of a convex set, Doklady Akademii Nauk SSSR (New Series) 59 (1948), 837-840 (Russian).

[4] F. E. Browder, Fixed-point theorems for noncompact mappings in Hilbert space, Proceedings of the National Academy of Sciences of the United States of America 53 (1965), 1272-1276.

[5] _ Nonexpansive nonlinear operators in a Banach space, Proceedings of the National Academy of Sciences of the United States of America 54 (1965), 1041-1044.

[6] _ Convergence of approximants to fixed points of nonexpansive non-linear mappings in Banach spaces, Archive for Rational Mechanics and Analysis 24 (1967), no. 1, 82-90.

[7] R. E. Bruck Jr., Nonexpansive retracts of Banach spaces, Bulletin of the American Mathematical Society 76 (1970), 384-386.

[8] _ Properties of fixed-point sets of nonexpansive mappings in Banach spaces, Transactions of the American Mathematical Society 179 (1973), 251-262.

[9] _ A common fixed point theorem for a commuting family of nonexpansive mappings, Pacific Journal of Mathematics 53 (1974), 59-71. 
[10] D. Göhde, Zum Prinzip der kontraktiven Abbildung, Mathematische Nachrichten 30 (1965), 251258.

[11] J.-P. Gossez and E. Lami Dozo, Some geometric properties related to the fixed point theory for nonexpansive mappings, Pacific Journal of Mathematics 40 (1972), 565-573.

[12] J. L. Kelley, General Topology, Van Nostrand Reinhold, New York, 1955.

[13] W. A. Kirk, A fixed point theorem for mappings which do not increase distances, The American Mathematical Monthly 72 (1965), 1004-1006.

[14] Z. Opial, Weak convergence of the sequence of successive approximations for nonexpansive mappings, Bulletin of the American Mathematical Society 73 (1967), 591-597.

[15] S. Reich, Asymptotic behavior of contractions in Banach spaces, Journal of Mathematical Analysis and Applications 44 (1973), no. 1, 57-70.

[16] _ Product formulas, nonlinear semigroups, and accretive operators, Journal of Functional Analysis 36 (1980), no. 2, 147-168.

[17] __ Strong convergence theorems for resolvents of accretive operators in Banach spaces, Journal of Mathematical Analysis and Applications 75 (1980), no. 1, 287-292.

[18] N. Shioji and W. Takahashi, Strong convergence theorems for asymptotically nonexpansive semigroups in Hilbert spaces, Nonlinear Analysis 34 (1998), no. 1, 87-99.

[19] T. Suzuki, On strong convergence to common fixed points of nonexpansive semigroups in Hilbert spaces, Proceedings of the American Mathematical Society 131 (2003), no. 7, 2133-2136.

[20] Strong convergence of Krasnoselskii and Mann's type sequences for one-parameter nonexpansive semigroups without Bochner integrals, Journal of Mathematical Analysis and Applications 305 (2005), no. 1, 227-239.

[21]__trong convergence theorems for infinite families of nonexpansive mappings in general Banach spaces, Fixed Point Theory and Applications 2005 (2005), no. 1, 103-123.

[22] _ The set of common fixed points of an n-parameter continuous semigroup of mappings, Nonlinear Analysis 63 (2005), no. 8, 1180-1190.

[23] _ The set of common fixed points of a one-parameter continuous semigroup of mappings is $F(T(1)) \cap F(T(\sqrt{2}))$, Proceedings of the American Mathematical Society 134 (2006), no. 3, 673-681.

[24]___ Browder's type convergence theorems for one-parameter semigroups of nonexpansive mappings in Banach spaces, to appear in Israel Journal of Mathematics.

[25] _ Browder's type convergence theorems for one-parameter semigroups of nonexpansive mappings in Hilbert spaces, to appear in Proceedings of the Fourth International Conference on Nonlinear Analysis and Convex Analysis (W. Takahashi and T. Tanaka, eds.), Yokohama Publishers, Yokohama.

[26] W. Takahashi and Y. Ueda, On Reich's strong convergence theorems for resolvents of accretive operators, Journal of Mathematical Analysis and Applications 104 (1984), no. 2, 546-553.

[27] B. Turett, A dual view of a theorem of Baillon, Nonlinear Analysis and Applications (St. Johns, Nfld., 1981), Lecture Notes in Pure and Appl. Math., vol. 80, Dekker, New York, 1982, pp. 279286.

Tomonari Suzuki: Department of Mathematics, Kyushu Institute of Technology, Sensuicho, Tobata, Kitakyushu 804-8550, Japan

E-mail address: suzuki-t@mns.kyutech.ac.jp 